\title{
Local Short Term Electricity Load Forecasting: Automatic Approaches
}

\author{
The-Hien Dang-Ha*, Filippo Maria Bianchi ${ }^{\dagger}$, Roland Olsson ${ }^{\ddagger}$ \\ * Department of Informatics, University of Oslo, Norway, Email: hthdang@student.matnat.uio.no \\ $\dagger$ Machine Learning Group, University of Tromsø, Norway, Email: filippo.m.bianchi@uit.no \\ $\ddagger$ Faculty of Computer Sciences Østfold University College, Østfold, Norway, Email: roland.olsson@ @iof.no
}

\begin{abstract}
Short-Term Load Forecasting (STLF) is a fundamental component in the efficient management of power systems, which has been studied intensively over the past 50 years. The emerging development of smart grid technologies is posing new challenges as well as opportunities to STLF. Load data, collected at higher geographical granularity and frequency through thousands of smart meters, allows us to build a more accurate local load forecasting model, which is essential for local optimization of power load through demand side management. With this paper, we show how several existing approaches for STLF are not applicable on local load forecasting, either because of long training time, unstable optimization process, or sensitivity to hyper-parameters. Accordingly, we select five models suitable for local STFL, which can be trained on different time-series with limited intervention from the user. The experiment, which consists of 40 time-series collected at different locations and aggregation levels, revealed that yearly pattern and temperature information are only useful for high aggregation level STLF. On local STLF task, the modified version of double seasonal HoltWinter proposed in this paper performs relatively well with only 3 months of training data, compared to more complex methods.
\end{abstract}

\section{INTRODUCTION}

Load forecasting is an integral part of electric power system operations, such as generation, transmission, distribution, and retail of electricity [18]. According to different forecast horizons and resolutions, load forecast problems can be grouped into 4 classes: long-term, mid-term, short-term and very shortterm. In this paper, we focus on Short-Term Load Forecast (STLF) of hourly electricity load for one day ahead.

Due to its fundamental role, STLF has been studied intensively over the past 50 years. However, the deployment of smart grid technologies brings new opportunities as well as challenges to the field. On a smart grid, load data can be collected at a much higher geographical granularity and frequency than before, by means of thousands of smart meters [18]. Such a larger availability of data allows the synthesis of a more local load forecasting model, which is essential to optimize power load locally in a demand-response paradigm [8]. We refer to a load time-series as "local" when it contains measurements relative to a small geographical region, whose average hourly load goes from several hundreds up to several hundreds of thousands of $\mathrm{kWh}$.

Despite the great variety of STLF methods proposed in the literature, most of them focus on load time-series relative to high aggregation levels (big towns, cities or entire countries), whose average goes from several to hundreds of MWh [13]. We found that these methods are not applicable to local STLF task for the following reasons:

- Long training time: unlike for STLF focusing on high aggregation level, in local STLF we need to train and update thousands of models at the same time. Predictions are made on hourly basis for many local regions, and the forecasting models must often be retrained (e.g., each month). This requirement cuts out approaches relying on slow derivative-free optimizers, such as evolutionary algorithms or particle swarm optimization [29], [30], [19].

- Unstable optimization process: since thousands of models needs to be trained at the same time, a local STLF model needs to be robust to the discrepancy in timeseries characteristics. For example, including long-term seasonal dependency into state space models makes their optimization process unstable.

- Sensitivity to hyperparameters: nonparametric techniques such as artificial neural networks and kernel estimation are characterized by a high sensitivity to the hyperparameters of the model. For example, FeedForward Neural Networks (FNN) have been proposed and extensively used for STLF since the 1990s [1], [16]. However, their prediction performance highly depends on the number of layers, the amount of nodes per layer, the regularization coefficients, and the learning rate. Such hyperparameters must be tuned through crossvalidation, which is time-consuming, due to the slow gradient descent training procedure, and does not guarantee convergence. Additionally, FNN approach requires a carefully-designed preprocessing such as outliers removal to work effectively [10], [15]. These problems make FNN unsuitable for the local STLF task. On the other hand, recurrent neural networks such as echo state networks and long short term memory networks are widely adopted in STLF [4]. However, these architectures are not considered in this work, as we focus on model-based approaches.

After having conducted a comprehensive survey of different STLF approaches, we selected five models, two of which are original variations of existing architectures, proposed in this paper for the first time. The models were chosen (or modified) to overcome the three aforementioned limitations and to be at the same time characterized by a high degree of automation, 
both in the training and in the prediction phase.

In our experiments, we process 40 time-series collected from separate locations and characterized by different aggregation levels. To the best of our knowledge, this is the first time a local STLF experiment has been done with such a large number of time-series with different characteristics. As expected, our experiments show that yearly patterns and temperature information are only useful for high aggregation level STLF. On very local load time-series (less than several hundreds of $\mathrm{kWh}$ ), the modified version of double seasonal Holt-Winter (modifiedDSHW) proposed in this paper performs relatively well with only 3 months of training data, compared to other more complex methods that require years of training data.

The remainder of the paper is organized as follows. In Section [II] the datasets under consideration are described, and the main characteristics of the load time-series are analyzed. The five proposed models and their origins are presented in Section III. Section IV explains the experiment setup and discusses the obtained results. Section $\mathrm{V}$ concludes the paper and suggests some future work.

\section{DATA DESCRIPTION}

The dataset under analysis consists of 40 load time-series collected from two countries, US and Norway, at different levels of aggregation. Such diversity in the data allows us to benchmark the generalization capability of various forecast methods. Among these 40 time-series, 20 of them come from the Global Energy Forecasting Competition 2012 (GEFCom2012). This dataset consists of 4 years of hourly load collected from a US utility with 20 zonal level series with average hourly load varies from $10.000 \mathrm{kWh}$ up to $200.000 \mathrm{kWh}$. The dataset is also accompanied by 11 temperature timeseries collected at the area, which can be used to improve the forecasting performance.

The other 20 load time-series come from Hvaler, a small island in Norway with around 6000 households. The island has been used as a smart grid pilot for many years, with over 8000 smart meters have been installed since 2012. The island power grid includes around 100 small distribution substations (including transformer on pole) organized hierarchically. Since there is currently no smart meters installed at these small substations, their loads are estimated by aggregating from their corresponding smart meters installed at households, street lights or other end consumers. The 20 time-series are relative to small distribution substations. They cover two years (20122013) and were selected based on the quality of data (e.g. the number of missing entries). As opposed to the GEFCom 2012 dataset, the Hvaler's load time-series are much more local and have less than $200 \mathrm{kWh}$ average hourly load. This allows us to test how different predictive models perform at various aggregation levels. Before delving into the details of each model, we first examine some characteristics of the load signals.

\section{A. Seasonal Patterns}

Fig. 1a shows the hourly load at Hvaler's substation 1 over 2 years. Through a simple inspection, we can observe a strong seasonal pattern characterized by high demand for electricity in winter and low demand in summer. This pattern evidences a dependency between weather conditions and power consumption. However, such a relationship depends also on geographic location and type of consumers. Fig. $1 \mathrm{~b}$ shows hourly load at zone 1 of the GEFCom 2012 dataset, indicating high demand during summer and winter, while low demand in other seasons. By analyzing the data more in detail, we can notice intraweek seasonal cycles (the load demand on the weekend is usually lower than on the weekdays) and intraday seasonal cycles, which arises from human routines (e.g. peaks at breakfast time and before dinner). Although these yearly, intraweek, and intraday seasonality effects are common in load timeseries, their importance is not studied in local STLF. In our experiment, we observed that the yearly pattern is useful only if the load time-series is highly aggregated.

\section{B. Weather Effects}

In load forecasting, weather conditions have always been an important factor. Although many meteorological elements like humidity, wind, rainfall, cloud cover, thunderstorm could be accounted for, the most influential and popular is the temperature, whose measurement is also easier to retrieve. In fact, temperature variables can explain more than $70 \%$ of the load variance in the GEF2012Com dataset [18] The scatter plot in Fig. 2 shows the relationship between load and temperature in both Hvaler and US. While the "V" shape is consistent with the two cases, there are still obvious differences on the relationship, which could be explained by the difference in geographical locations, human comfortable temperature, heating/cooling technology, or type of consumers (e.g. industrial or residential units).

\section{Calendar Effects}

People change their daily routines on calendar events, such as holidays, festivities and special events (e.g. football matches, transportation strike), with a possible modification in the electricity demand. Those situations represent outliers and could be treated differently to improve the model accuracy. In our study, only the national holiday events are taken into account, due to the lack of information on other events.

\section{Long-Term Trend}

The scale, variation, and other properties of the load signal could change over time due to changes in population, technology, or economic conditions. In Fig. 1, we can see a tendency of increasing consumption over the years. In our experiment, two methods explicitly model and detrend the load time-series in the first step. Other methods either model it implicitly or ignore it. This is because the long-term trend can be considered as constant in short-term and may not contribute much to oneday-ahead load forecasting. 


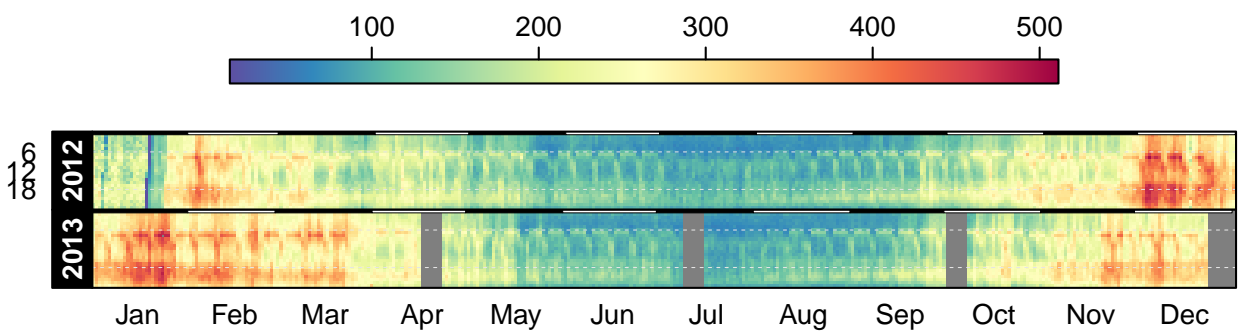

(a) Load profile of substation 1 from Hvaler with the 4 testing periods are masked out.
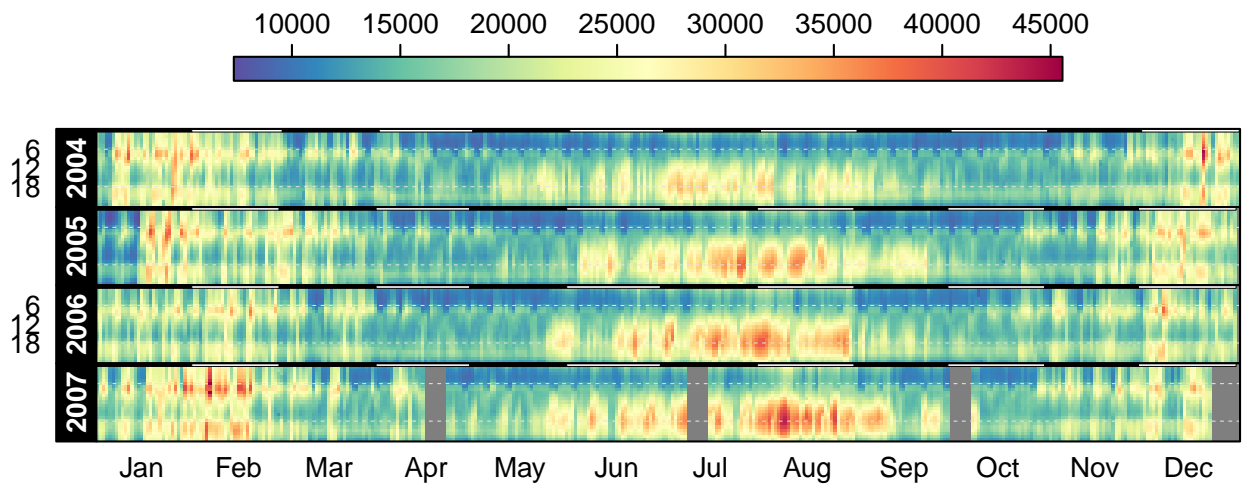

(b) Load profile of zone 1 from GEFCOM2012 (in $\mathrm{kWh}$ ) with the 4 testing periods are masked out.

Fig. 1. Hourly load profiles in KWh of the substation 1 from Hvaler (top) and zone 1 from GEFCom2012 dataset (bottom).

\section{Methodology}

In this section, we review several important STLF approaches. We divided the STLF methodologies of interest into four main categories: Averaging, Linear State Space, Decomposition, and Data-Driven approaches. This classification is particularly meaningful for our analysis, but other taxonomies have been proposed in the literature [18], [1], [2]. For each category we provide a short overview, pointing out the main advantages and disadvantages encountered in our applicative scenario. Based on this analysis, one baseline and five potential solutions were implemented and tested on the Hvaler and GEFCom2012 data.

\section{A. Averaging Approach}

Despite being very basic, this is still a popularly used method due to its simple implementation and obvious model interpretation [9]. The averaging model makes predictions based on linear combinations of consumption values from "similar" days and was used as a benchmark in [28]. The forecast is computed as

$$
\hat{y}_{t}(k)=\frac{y_{t+k-s_{2}}+y_{t+k-2 s_{2}}+y_{t+k-3 s_{2}}+y_{t+k-4 s_{2}}}{4},
$$

where $y_{t}$ is the demand in period $t, k$ is the forecast lead time, and $s_{2}$ is the so called second seasonal cycle, which is the intraweek $s_{2}=24 * 7=168$. The model predicts future load by averaging the corresponding observations in each of the previous four weeks. As previously discussed, there are 3 typical seasonal cycles in load time-series, which are intraday, intraweek and yearly cycles. In this paper, their cycle lengths (in hour) are represented respectively as $s_{1}=24, s_{2}=24 * 7=$ 168 , and $s_{3}=24 * 364.25=8766$. Note that we fixed the length of each seasonal cycle and did not re-estimate it in different time-series. This is because the estimation requires analyzing a periodogram manually and is hard to automate.

At first, we intended to use the averaging model as the baseline to estimate the forecast difficulty. However, the model produced highly autocorrelated errors in the residual series and could not serve as a good baseline. Therefore, we decided to train an additional ARIMA model on the residual series produced by the averaging model. We call this the avgARIMA model and use it as the baseline model in our experiment.

\section{B. Linear State Space Approach}

State space approach refers to those models that can be written in a linear state space form, which consists of a set of states with initial distribution (usually Gaussian), a measurement equation and a Markovian transition equation. Although state space models can be extended to include exogenous variables, such as temperature, the univariate setting is still the most popular in STLF. Recent studies have shown that, although in the long run the load is strongly influenced by meteorological conditions and special events, an univariate model is sufficient in shorter lead times [27]. The two most common and accurate state space models that have been reported for STLF task in the literature are Auto-Regressive Integrated Moving-Average (ARIMA) and Holt-Winters exponential smoothing.

1) ARIMA: The ARIMA model was adopted in STLF back in 1987 [14], where a double seasonal ARIMA model 


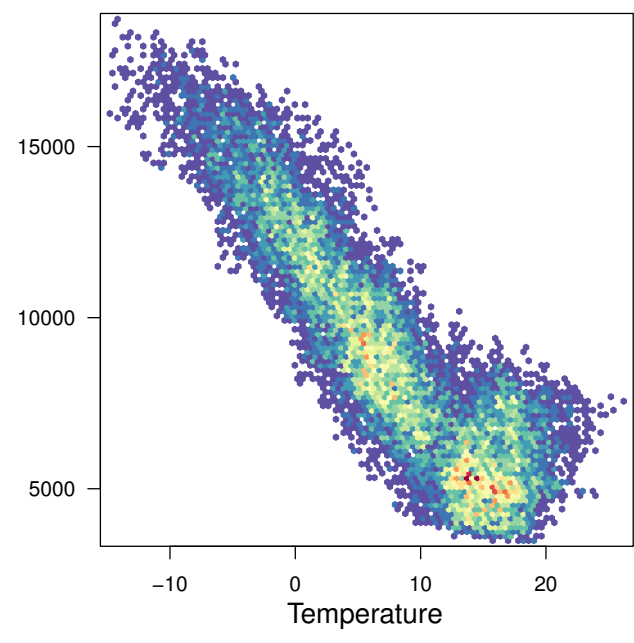

(a) Hvaler - Norway
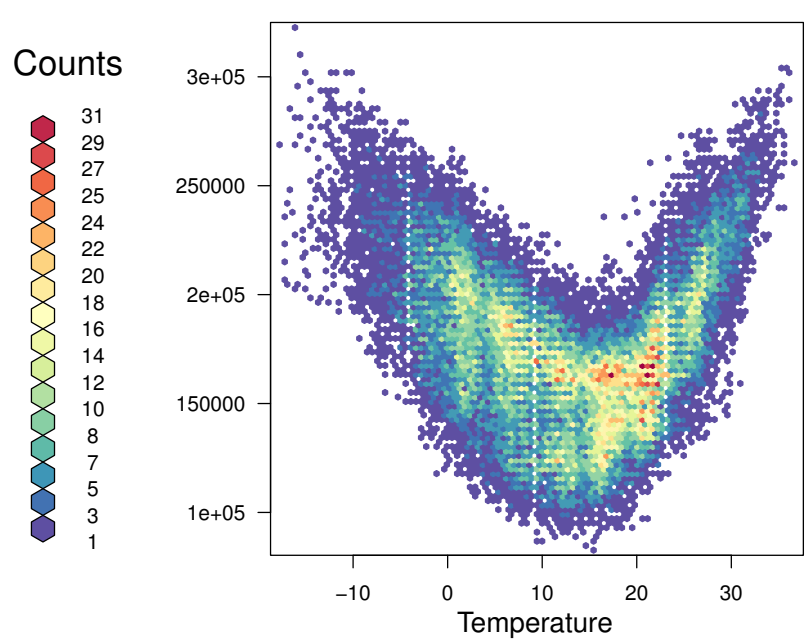

(b) GEFCom2012 - US
Counts

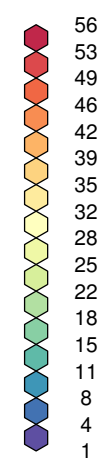

Fig. 2. Relationship between Temperature $\left({ }^{\circ} \mathrm{C}\right)$ and Consumption (KWh) in Hvaler (top) and GEFCom2012 dataset (bottom) was experimented (intraday and intraweek cycles). This approach remains popular, with extensions to include exogenous variables, or intrayear seasonal cycles [29], [26]. One big disadvantage of ARIMA is that the model hyperparameters (such as AR, I, and MA orders, as well as orders of seasonal AR, I, and MA terms) are usually derived from the BoxJenkins test, which is hard to automate and still require human expertise to examine the partial correlogram of the timeseries [23], [2]. These hyperparameters can be heuristically fixed a-priori [28], [26], However, during our experiment we noted that in the ARIMA model with (double) seasonality the optimization process becomes highly unstable when fixing the AR, I, MA orders and all the seasonal AR, I and MA orders to some arbitrary numbers. Akaike Information Criterion can also be used to set ARIMA hyperparameters. However, a complete search over all possible models is time-consuming, especially for seasonal ARIMA. Therefore, in this paper, we only use ARIMA to correct the autocorrelation in the residual series produced by other models. This is a common practice in timeseries forecasting to improve accuracy when the main model has autocorrelated errors.

2) Holt-Winters: Holt-Winters is another popular state space model that accommodates the two intraday and intraweek seasonal cycles that commonly appear in load timeseries. Taylor et.al. (2003) [25] introduced the double seasonal Holt-Winters method (DSHW), whose important advantage, which we find suitable for our local load forecasting problem, is that it only requires the length of the two seasonal cycles to be specified. We indeed did not get any optimization problem when fixing these two seasonal cycles to the intraday $s_{1}=24$ hours and intraweek $s_{2}=168$ hours. Implementation details are provided in Section IV] where we also introduce a modified version of the original DSHW that yields significantly better performance in our experiment.

In 2010, Taylor et.al. [26] proposed the triple seasonal HoltWinters (TSHW), which enables the model to accommodate the intrayear seasonal cycles. However, it turned out that the extra seasonality causes the training process to become much slower and unstable. In fact, the success of the optimization process depends on the choice of initial values for the initial states. To address this issue, Taylor et.al. generated $10^{4}$ initial vectors as possible initialization for the variables of the model. Since this process requires significant computational power, the TSHW is unsuitable for the local STLF task.

\section{Data-Driven Approach}

Instead of modeling the underlying physical processes, data-driven methods try to discover consistent patterns from historical data, according to a machine learning approach. A mapping between the input variables and the load is learned and then used for prediction. Depending on the forecasting task, the input and output variables are designed accordingly. In the following, we review two of the main approaches in STLF.

1) Nonlinear Non Auto-Regressive Regression: This approach models the load as a non-linear function of only exogenous input variables without using the autoregressive terms. According to the nature of the data discussed in Section [II. potential exogenous variables of interest are: time of day, time within week, time of year, linear trend, temperatures, or smoothed temperatures. Frameworks such as random forests and gradient boosted model... can be used to map inputs to the desired output. However, since these approaches do not explicitly model the autocorrelation that almost always exists in the load time-series, they must be used in combination with other techniques to be effective, such as a state space and a long-term decomposition model [21]. This requires extra effort on the model deployment and management process. For this reason, we did not include this approach in our study.

2) Nonlinear Auto-Regressive with Exogenous (NARX): An NARX model computes the next value of a variable, from 
previous values of the variable itself and current and past values of exogenous series. The basic formulation reads as

$$
y_{t}=F\left(y_{t-1}, y_{t-2}, \ldots, x_{t}, x_{t-1}, x_{t-2}, \ldots\right),
$$

where $F(\cdot)$ is a non-linear function, which could be modeled by any general-purpose machine learning model such as artificial neural network (ANN) or support vector machine (SVM).

As discussed in Section I. ANN depends on several hyperparameters and its training can be cumbersome. An ANN is prone to overfitting and is sensitive to outliers [18]. The problem could be solved by replacing the ANN with a model characterized by a lower variance such as SVM, which has been adopted in several studies [7], [3], [24].

A different approach is to use the random forest (RF) as in [5]. Thanks to its bagging, data sub-sampling, and random features selection process, the RF model is capable of capturing complex patterns, while maintaining a low variance [20]. This approach is called $N A R X-R F$ and is specified in Section IV-B

\section{Time-Series Decomposition Approach}

Time-series decomposition approach deconstructs a timeseries into several components, each component represents different kinds of pattern. According to the nature of load timeseries discussed in Section II] potential components are longterm trend, intrayear cycle, intraweek cycle, intraday cycle, relationship between temperature and load, holiday events, ...

In GEFCom2012, Lloyd and James (2014) [21] used a Gaussian Process to decompose the load time-series. Their Gaussian Process contains a set of different kernels, each of them is designed to captured different component in the time-series. The long-term trend is captured by a squared exponential kernel, intrayear cycle is captured by a periodic kernel of time, while the relationship between temperature and load is captured by a squared exponential kernel of temperature. Although this hybrid approach works relatively well on GEFCom2012 data, a GP model needs to be manually carefully designed and requires special treatment on different load signals [21]. Therefore, we found Gaussian Process not suitable for the local STLF task and did not include it in the experiment.

Another popular way to decompose a load time-series is to use linear additive models, where the load is modeled as a linear combination of various independent features. The learned model is interpretable, easy to implement/automate the training process, and able to achieve high accuracy. Many different features have been suggested in the literature to capture different load components. For example, the yearly cycle can be modeled by 8 Fourier series [12] or spline functions [22]; relationship between temperature and consumption can be modeled by a pice-wise linear [12], quadratic [6], or spline functions [22]; monthly change in relationship between temperature and consumption can be modelled by interaction terms between temperature and month of year variable [17]. Among different linear additive models proposed in the literature, we found that the TBATS model suggested by
De Livera et. al. 2011 [11] and the semi-parametric additive model suggested by Goude et.al. 2014 [22] are the two most suitable models for the local STLF task.

The name TBATS is an acronym for key features of the model: Box-Cox transforms, ARMA errors, Trend, and Trigonometric Seasonal components. On the other hand, the semi-parametric additive approach bases on the Generalized Addictive Model. Precise specification of these two models are given in Section IV-B

\section{EXPERIMENTS AND DISCUSSION}

\section{A. Experiment Setting}

In this section, we show experiment results of the five chosen models on 40 time-series described in section II Each time-series is marked with 4 testing periods, which are the four following weeks of the final year data: 16, $28,40,52,53$ (note that week 53 contains just 1 day or no day at all). These weeks were chosen to bring a fair estimation of model performance in different seasons and holidays during the final week of the year. These testing periods are demonstrated in Fig. 1. For each testing period, we did multi-step rolling forecasts without re-estimation. For different testing period, we re-estimated the model. We did not re-estimate the models within one testing period since this approach is impractical. Retraining and updating thousands of models every hour or even every day are infeasible since they require a tremendous amount of computing resource. We used the Mean Average Percentage Error (MAPE) to compare model performance, which is widely used in the energy forecasting community. The MAPEs are calculated separately at 24 prediction horizons for 24 hours ahead. Besides accuracy, we also report the training time of each model, which is an important factor in deciding which model to use in practice but rarely mentioned in the literature. Precise model specifications of one baseline and all five chosen methods are given in the following section. The whole experiment can be easily reproduced from the data and code publicly available at: https://github.com/Nikasa1889/R_Notebooks

\section{B. Model Specifications}

1) avgARIMA: The avgARIMA model was used to give a baseline performance for our experiment. First, an averaging model is used to predict the future load by averaging the corresponding observations in each of the previous four weeks, as specified in (1). Its 3-month residual time series is then used to train an ARIMA. A stepwise search was used to optimize the AR, I and MA orders. The procedure is implemented by the auto.arima() function in the $\mathrm{R}$ forecast package.

2) originalDSHW and modifiedDSHW: The multiplicative formulation for the original double seasonal Holt-Winders model $(D S H W)$ is given in the following expression [25], [28]:

$$
\begin{gathered}
l_{t}=\alpha\left(y_{t} /\left(d_{t-s_{1}} w_{t-s_{2}}\right)\right)+(1-\alpha) l_{t-1} \\
d_{t}=\theta\left(y_{t} /\left(l_{t} w_{t-s_{2}}\right)\right)+(1-\theta) d_{t-s_{1}} \\
w_{t}=\omega\left(y_{t} /\left(l_{t} d_{t-s_{1}}\right)\right)+(1-\omega) w_{t-s_{2}}
\end{gathered}
$$


$\hat{y}_{t}(k)=l_{t} d_{t-s_{1}+k} w_{t-s_{2}+k}+\phi^{k}\left(y_{t}-\left(l_{t-1} d_{t-s_{1}} w_{t-s_{2}}\right)\right)$

, where $l_{t}$ is the smoothed level; $d_{t}$ and $w_{t}$ are the seasonal indices for the intraday and intraweek seasonal cycles, respectively; $\alpha, \theta$, and $\omega$ are the smoothing parameters. The term involving the parameter $\phi$ in the forecast equation (6) is a simple adjustment for first-order autocorrelation. This model is implemented in the R forecast package under the name $d s h w($ ), and named origDSHW in this paper.

During the experiment, we recognized that performance of the origDSHW model can be improved significantly if we employ a different objective function. Instead of using the sum of square errors of the in-sample 1-hour ahead forecast, we modified the objective function to the sum of squared errors of all 24 horizons in-sample forecast. Moreover, we also increased the upper limit of the $\phi$ parameter from 0.9 to 0.99 . This model is called modDSHW in this experiment. Both the origDSHW and modDSHW models were trained on 3 months of data.

3) NARX-RF: Although an NARX model with SVM yielded good performance in other studies, we found it hard to automate, since its performance depends heavily on the choice of hyper-parameters: the cost of errors $C$ and width of the $\epsilon$-insensitive tube. Moreover, optimal values of these hyper-parameters vary very much on different load signals. Therefore, instead of SVM, the random forest was used, which is referred to as the $N A R X-R F$ model in this paper. For building the random forest, the package ranger in $\mathrm{R}$ was used.

To avoid multi-step ahead predictions, a separate random forest was used for each lead time. For lead time $h$, the set of inputs consists of the load values at lags: $1,2,3, s_{1}-h$, $2 s_{1}-h, 3 s_{1}-h, s_{2}-h, 2 s_{2}-h$; two temperature-related exogenous variables: temperature and exponential smoothed temperature; and three calendar variables: time of day and day of week. The smoothed temperature is often used in STLF to take into account the physical inertia of buildings and delay effects of temperature on consumption [12]. The coefficient for the temperature exponential smoothing process was set to 0.85 .

We kept all the default settings in the ranger function, which set the number of trees to grow ntree $=500$, and the number of candidate features at each split mtry $=3$. The subsampling ratio was set so that each tree receives 5000 data points to train on. The model makes use of all the available data up to the testing point.

4) TBATS: The TBATS model was introduced by De Livera et.al. in 2011 [11] to solve the forecasting problem in time series with complex seasonal patterns such as multiple seasonal periods or high-frequency seasonality. The model incorporates Box-Cox transformations, linear trend, Fourier representations with time-varying coefficients, and ARMA error correction. The method involves a simple, yet efficient estimation procedure, which makes it suitable for the local STLF problem. In this experiment, the exact TBATS model described in [11] was used without any modification. The
TBATS implementation provided in the forecast package was used with all the default settings unchanged.

5) SemiParametric: The semi-parametric additive model was first introduced by Goude et.al. in the GEFCom 2012 competition [22]. In 2014, Goude et.al. have tested the method's generalization ability, where it was used for short and medium-term load forecasting on 2206 large-scale substations automatically [13]. Here we present a short explanation of the method, together with some small adaptation we have done to make it more appropriate for the local STLF task. For short, this method is called SemiPar in this paper.

The SemiPar method splits the load into three parts:

$$
Z_{t}=Z_{t}^{l t}+Z_{t}^{m t}+Z_{t}^{s t},
$$

where $Z_{t}$ is the electrical load at time $t, Z_{t}^{l t}$ is the long-term part of the load, corresponding to low-frequency variations such as long-term trends or economic effects. $Z_{t}^{m t}$ is the medium-term part, incorporating daily to weekly effects, the meteorological effects, and the calendar effects. The short term part, $Z_{t}^{s t}$, contains everything that could not be captured on a large temporal scale but could be obtained locally in time. We implemented the $Z_{t}^{l t}$ and $Z_{t}^{m t}$ exactly the same as described in [22]. However, for the short term $Z_{t}^{\text {st }}$ part, we use an ARIMA model (optimized by the auto.arima() function) to capture the auto-correlation in the residual time-series after removing the long-term and medium-term parts.

The long-term forecast uses combination of generalized additive models (GAM) and kernel regression, while the medium-term forecast uses GAMs. The GAM model with generalized cross validation criterion is implemented in the $\mathrm{R}$ package $m g c v$, while the kernel regression is the NadarayaWatson model, which is available through the bbemkr package.

For the long-term model, we aggregate the consumption and temperature by month, denoted by $Z_{t}^{\text {monthly }}$ and $T_{t}^{\text {monthly }}$. Then we estimate monthly consumption using the following semi-parametric additive model [22]:

$$
\hat{Z}_{t}^{\text {monthly }}=\sum_{q=1}^{12} c_{q} I_{\text {Month }_{t}=q}+f\left(T_{t}^{\text {monthly }}\right)+\epsilon_{t}
$$

Where:

- $I_{\text {Month }_{t}=q}$ is an indicator variable which is equal to 1 when the month at observation $t$ is $q$ (from 1 to 12), and 0 otherwise.

- $f$ is the effect of the monthly temperature, estimated by thin plate regression splines (default setting in $m g c v$ package).

The monthly estimated residuals are then obtained as follows:

$$
\hat{\epsilon}^{\text {monthly }}=Z_{t}^{\text {monthly }}-\hat{Z}_{t}^{\text {monthly }}
$$

Then the residuals are smoothed and interpolated to hourly frequency by using Nadaraya-Watson kernel regressors, with Gaussian kernels and a bandwidth of 12 . These smoothed residuals are a good estimate of low-frequency effects, which contains neither annual seasonality nor weather effects. These 
residuals are considered as $Z_{t}^{l t}$ and smooth by construction, and thus they are constantly extrapolated for one-day horizon.

By removing $Z_{t}^{l t}$ from the original load, we get the signal $Z_{t}^{\text {det }}$ which contains $Z_{t}^{m t}$ and $Z_{t}^{\text {st }}$. We fit one mid-term model for each hour of the day so that we have 24 mid-term models. These mid-term models are GAM in the following form [22]:

$$
\begin{array}{r}
Z_{t}^{\text {det }}=\sum m_{q} I_{\text {Day }_{\text {Type }}=q}+ \\
+g_{1}\left(\theta_{t}\right)+g_{2}\left(T_{t}\right) \\
+h\left(\text { toy }_{t}\right)+\epsilon_{t}
\end{array}
$$

where:

- $Z_{t}^{\text {det }}$ is the de-trended electrical demand at time $t$.

- DayType $t_{t}$ is type of day for observation $t .1$ for Sunday, 2 for Monday, 3 for Tuesday, 4 for Wednesday, 5 for Thursday, 6 for Friday, 7 for Saturday, 8 for Christmas and New Years Day, 9 for Christmas Eve, 10 for Independence Day, and 11 for Thanksgiving.

- $\theta_{t}$ is the smoothed temperature, obtained via exponential smoothing of the real temperature $T_{t}: \theta_{t}=(1-0.85) T_{t}+$ $0.85 \theta_{t-1}$

- toy $_{t}$ is the time of year, which is the position of the observation $t$ within the year. $h\left(\right.$ toy $\left._{t}\right)$ corresponds to the smooth yearly cycle of the load.

- All the $g($.$) functions are modeled by thin plate regression$ splines, while the $h($.$) function is modeled by cyclic cubic$ regression splines.

An ARIMA short term model is then built to capture patterns in the residuals after removing $Z_{t}^{l t}$ and $Z_{t}^{m t}$ from $Z_{t}$.

\section{Experiment Results}

The whole experiment was trained on an Intel Core i7$6700 \mathrm{k} 4.0 \mathrm{Ghz}$ machine with 8 cores. Training time of each method is reported in Fig. 3, where the CPU time was measured for one core. The whole experiment took about 12 hours to complete.

Fig. 4 shows a comparison between median MAPE of each method at different prediction horizons on the two datasets GEFCom2012 and Hvaler. On the GEFCom2012 dataset, the SemiPar method is obviously the best model. This is expected since it is the only model that has been tested and performed well in a large-scale experiment with thousands of timeseries. It is also the only model that explicitly captures all the patterns discussed in section II including the long-term, midterm, and the short-term patterns together with the temperature effect. However, on the Hvaler dataset, where all the loads are collected at a much lower aggregation level, the SemiPar method exhibits its limitation. It performs only slightly better than the NARX-RF approach in the first ten horizons and then becomes worse when the prediction horizon increases. This can be explained by the fact that the load time-series in Hvaler are much noisier than in GEFCom2012 dataset since they consist of only a small number of consumers. Therefore, their long-term and mid-term trends are less consistent in the long run, which causes the decomposition approach to be less effective. The load in Hvaler is also collected in a shorter

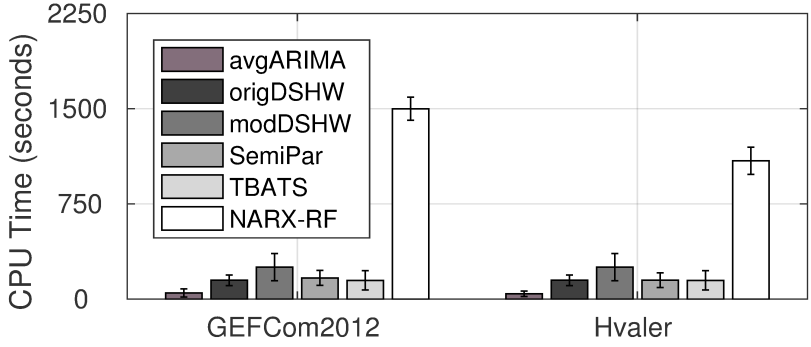

Fig. 3. Training time of each method running on an Intel core i7-6700k 4.0Ghz 8 cores. The $\mathrm{CPU}$ time was measured for one core.

period ( 2 years), which causes the estimation of the long-term and mid-term components to become less accurate. On the other hand, the short-term processes like intra-week, intra-day, and innovations become more influential in Hvaler dataset. This explains why the modDSHW method, which only uses 3 months of training data and only models the intra-week and intra-day seasonality, can slightly outperform the SemiPar at horizons further than 10 .

The second best model in both of the two GEFCom 2012 and Hvaler datasets is NARX-RF. It performs consistently well on the two datasets at all the prediction horizons, only significantly worse than SemiPar for early horizons. This consistency in performance is an important advantage if the system contains load time-series collected from many different scales, and we want to use only one forecasting method to simplify the deployment process. However, one has to consider its running time, since NARX-RF is more than one order of magnitude slower than other methods.

Our modifications made for the DSHW model turn out to be very effective. The modDSHW method significantly outperforms the orgiDSHW method in every case. This suggests that one should always follow these modifications if the DSHW model is of his interest. The modDSHW performs surprisingly well on the Hvaler dataset, even without using temperature information and was trained in only 3 months of data. Therefore, we believe that temperature information only contributes marginally to the forecasting accuracy on very local load time-series like Hvaler.

The TBATS method performs badly on both datasets and even worse than the averageARIMA model at some points. This can be because the way it decomposes the time-series is not suitable for the load signal.

\section{Conclusions and Future Work}

In this paper, we were looking for solutions for local one-day-ahead load forecasting problem, which needs to be able to model thousands of load time-series automatically without human intervention. One baseline and five models have been proposed, including avgARIMA, orgiDSHW, modDSHW, NARX-RF, TBATS, and SemiPar. These models were tested on 40 different load time-series, collected from US and Norway at different aggregation levels with different characteristics. The experiment results show that the SemiPar has superior performance on high-aggregation load, at the 


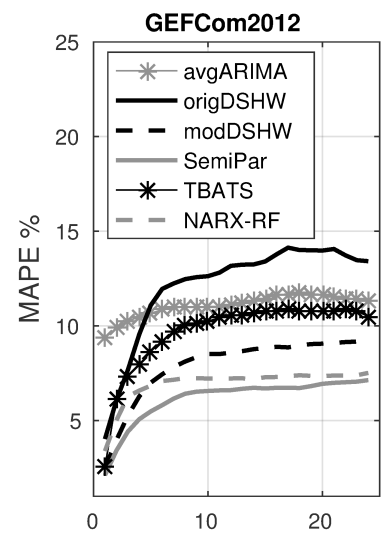

Forecast Horizon (hours)

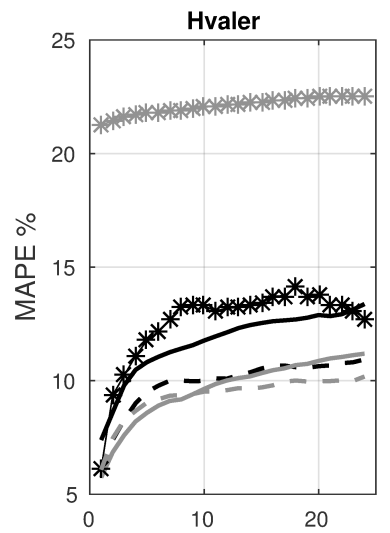

Forecast Horizon (hours)
Fig. 4. Comparison between median MAPE of each method at different prediction horizons on GEFCOM2012 (left) and Hvaler (right)

cost of a long historical data requirement. On the other hand, NARX-RF performs consistently well in many cases, at the expense of long training time. On low-aggregation load time-series, our modified version of the DSHW model works surprisingly well with only 3 months of training data and without using temperature information. If the historical data is limited, which is the case when a new smart grid is installed, the modDSHW model is highly recommended. The experiment also suggests that at low aggregation level, long term underlying processes (e.g., trend or intra-year cycle) and temperature information do not contribute much to the forecasting accuracy. Apparently, one can develop a better and more general model for the task by automatically combine or select among those methods proposed in this paper. However, one must acknowledge the fact that this would complicate the deployment and maintenance process, where thousands of models are involved.

\section{REFERENCES}

[1] Hesham K. Alfares and Mohammad Nazeeruddin. Electric load forecasting: Literature survey and classification of methods. International Journal of Systems Science, 33(1):23-34, 2002.

[2] S Aman, M Frincu, C Charalampos, U Noor, Y Simmhan, and V Prasanna. Empirical comparison of prediction methods for electricity consumption forecasting. University of Southern California, Tech. Rep, pages 14-942, 2014.

[3] Zeyar Aung, Mohamed Toukhy, John Williams, Abel Sanchez, and Sergio Herrero. Towards accurate electricity load forecasting in smart grids. In DBKDA 2012, The Fourth International Conference on Advances in Databases, Knowledge, and Data Applications, pages 5157, 2012.

[4] F. M. Bianchi, E. De Santis, A. Rizzi, and A. Sadeghian. Short-term electric load forecasting using echo state networks and pca decomposition. IEEE Access, 3:1931-1943, 2015.

[5] Leo Breiman. Random forests. Machine learning, 45(1):5-32, 2001.

[6] Nathaniel Charlton and Colin Singleton. A refined parametric model for short term load forecasting. International Journal of Forecasting, 30(2):364-368, 2014.

[7] Bo-Juen Chen, Ming-Wei Chang, and Chih-Jen Lin. Load forecasting using support vector machines: A study on eunite competition 2001. Power Systems, IEEE Transactions on, 19(4):1821-1830, 2004.

[8] Albert Chiu, Ali Ipakchi, Angela Chuang, Bin Qiu, D Brooks, E Koch, J Zhou, MK Zientara, PR Precht, R Burke, et al. Framework for integrated demand response (dr) and distributed energy resources (der) models. NAESB \& UCAIug. September, 2009.

[9] Katie Coughlin, Mary Ann Piette, Charles Goldman, and Sila Kiliccote. Estimating demand response load impacts: evaluation of baseline load models for non-residential buildings in california. Lawrence Berkeley National Laboratory, 2008.

[10] APA da Silva, AJ Rocha Reis, MA El-Sharkawi, and RJ Marks. Enhancing neural network based load forecasting via preprocessing. In Proceedings of the International Conference on Intelligent System Application to Power Systems, Budapest, Hungary, pages 118-123, 2001.

[11] Alysha M De Livera, Rob J Hyndman, and Ralph D Snyder. Forecasting time series with complex seasonal patterns using exponential smoothing. Journal of the American Statistical Association, 106(496):1513-1527, 2011.

[12] V. Dordonnat, S. J. Koopman, M. Ooms, a. Dessertaine, and J. Collet. An hourly periodic state space model for modelling French national electricity load. International Journal of Forecasting, 24(4):566-587, 2008.

[13] Yannig Goude, Raphael Nedellec, and Nicolas Kong. Local short and middle term electricity load forecasting with semi-parametric additive models. IEEE Transactions on Smart Grid, 5(1):440-446, 2014.

[14] Martin T Hagan and Suzanne M Behr. The time series approach to short term load forecasting. Power Systems, IEEE Transactions on Power Systems, 2(3):785-791, 1987.

[15] Luis Hernandez, Carlos Baladrón, Javier M Aguiar, Belén Carro, Antonio J Sanchez-Esguevillas, and Jaime Lloret. Short-term load forecasting for microgrids based on artificial neural networks. Energies, 6(3):13851408, 2013

[16] Tao Hong. Short term electric load forecasting. Phd thesis, North Carolina State University, 2010.

[17] Tao Hong, Min Gui, Mesut E. Baran, and H. Lee Willis. Modeling and forecasting hourly electric load by multiple linear regression with interactions. IEEE PES General Meeting, PES 2010, pages 1-8, 2010.

[18] Tao Hong and Mohammad Shahidehpour. Load Forecasting Case Study. National Association of Regulatory Commissioners, pages 1-171, 2015.

[19] Gwo-Ching Liao and Ta-Peng Tsao. Application of a fuzzy neural network combined with a chaos genetic algorithm and simulated annealing to short-term load forecasting. IEEE Transactions on Evolutionary Computation, 10(3):330-340, 2006.

[20] Andy Liaw and Matthew Wiener. Classification and regression by randomforest. $R$ news, 2(3):18-22, 2002.

[21] James Robert Lloyd. GEFCom2012 hierarchical load forecasting: Gradient boosting machines and Gaussian processes. International Journal of Forecasting, 30:369-374, 2014.

[22] Raphael Nedellec, Jairo Cugliari, and Yannig Goude. GEFCom2012: Electric load forecasting and backcasting with semi-parametric models. International Journal of Forecasting, 30(2):375-381, 2014.

[23] Theresa Hoang Diem Ngo Ngo. The Box-Jenkins Methodology for Time Series Models. Proceedings of the SAS Global Forum 2013 conference, 6:1-11, 2013.

[24] N. Sapankevych and Ravi Sankar. Time Series Prediction Using Support Vector Machines: A Survey. IEEE Computational Intelligence Magazine, 4(2):24-38, 2009.

[25] James W Taylor. Short-term electricity demand forecasting using double seasonal exponential smoothing. Journal of the Operational Research Society, 54(8):799-805, 2003.

[26] James W. Taylor. Triple seasonal methods for short-term electricity demand forecasting. European Journal of Operational Research, 204(1):139-152, 2010.

[27] James W. Taylor, Lilian M. de Menezes, and Patrick E. McSharry. A comparison of univariate methods for forecasting electricity demand up to a day ahead. International Journal of Forecasting, 22(1):1-16, 2006.

[28] James W Taylor, Patrick E Mcsharry, and Senior Member. Short-Term Load Forecasting Methods : An Evaluation Based on European Data. 22(4):2213-2219, 2007.

[29] Bo Wang, Neng-ling Tai, Hai-qing Zhai, Jian Ye, Jia-dong Zhu, and Liang-bo Qi. A new armax model based on evolutionary algorithm and particle swarm optimization for short-term load forecasting. Electric Power Systems Research, 78(10):1679-1685, 2008.

[30] Chih-Hung Wu, Gwo-Hshiung Tzeng, and Rong-Ho Lin. A novel hybrid genetic algorithm for kernel function and parameter optimization in support vector regression. Expert Systems with Applications, 36(3):47254735, 2009 